\title{
A General Maximum Progression Model to Concurrently Synchronize Left-Turn and through Traffic Flows on an Arterial
}

\author{
Binbin Jing (iD) and Jianmin Xu \\ School of Civil Engineering and Transportation, South China University of Technology, Guangzhou, Guangdong 510640, China \\ Correspondence should be addressed to Binbin Jing; jingbin19@126.com
}

Received 10 October 2017; Revised 8 January 2018; Accepted 21 January 2018; Published 21 February 2018

Academic Editor: Luca D’Acierno

Copyright (C) 2018 Binbin Jing and Jianmin Xu. This is an open access article distributed under the Creative Commons Attribution License, which permits unrestricted use, distribution, and reproduction in any medium, provided the original work is properly cited.

In the existing bandwidth-based methods, through traffic flows are considered as the coordination objects and offered progression bands accordingly. However, at certain times or nodes in the road network, when the left-turn traffic flows have a higher priority than the through traffic flows, it would be inappropriate to still provide the progression bands to the through traffic flows; the left-turn traffic flows should instead be considered as the coordination objects to potentially achieve better control. Considering this, a general maximum progression model to concurrently synchronize left-turn and through traffic flows is established by using a time-space diagram. The general model can deal with all the patterns of the left-turn phases by introducing two new binary variables into the constraints; that is, these variables allow all the patterns of the left-turn phases to deal with a single formulation. By using the measures of effectiveness (average delay time, average vehicle stops, and average travel time) acquired by a traffic simulation software, VISSIM, the validity of the general model is verified. The results show that, compared with the MULTIBAND, the proposed general model can effectively reduce the delay time, vehicle stops, and travel time and, thus, achieve better traffic control.

\section{Introduction}

As an important approach applied in urban traffic signal control, arterial traffic signal coordination has tremendous importance in reducing the vehicle delay time and vehicle stops, improving the passing efficiency of arterials and alleviating the traffic congestion. Studies on arterial traffic signal coordination can be divided into two types based on the different optimization objectives. The first type aims to minimize the delay time, whereas the second type aims to maximize the progression bandwidths. A delay-based method establishes a relational expression between the delay time and traffic signal parameter combinations (such as cycle length, split, and offset) and calculates the delay time for different signal parameter combinations, thereby obtaining the optimal signal parameter combinations with a minimum delay time by comparison. A bandwidth-based method, accordingly, aims to maximize the two-way progression bandwidths of an arterial, so that the vehicles, when driving within the progression bands, can pass through the entire arterial without any stops. A bandwidth-based method is the preferred choice of numerous traffic engineers because it can visually display the coordination control effects. Morgan and Little are pioneers who studied the problem of arterial traffic signal coordination and formulated the concept of bandwidths [1]. Subsequently, Little extended their previous work and established a mixed-integer linear programming model for arterial traffic signal coordination by considering the maximum two-way progression bandwidths as the objective functions and cycle length, offset, and progression speed as the decision variables [2]. Messer et al. developed a traffic signal progression program to optimize multiphase sequences to obtain the maximum progression bandwidths [3]. In 1981, Little et al. further extended their previous research by considering issues such as queue clearance time and left-turn phase sequence and proposed a more universal coordination model-the MAXBAND model [4]. Chang et al. extended the research by Little and developed the MAXBAND-86 model [5]. In comparison with the MAXBAND model, the MAXBAND-86 model can solve the traffic signal coordination problem of a closed road network enclosed by multiple 
arterials. A progression bandwidth may not be realized or be only partially realized when the signal timings generated by the MAXBAND model and PASSER II are actually applied to arterials. Tsay and Lin proposed a new algorithm for solving the maximum progression bandwidth to ensure that the users could receive a more realistic range [6]. In actual situations, different road sections have different traffic and saturation volumes, and so, their demands for the progression bandwidths also differ. Therefore, it is preferable to assign different progression bandwidths to different road sections to better satisfy the actual traffic requirements. Gartner et al. took this into consideration and proposed a variable progression bandwidth model-the MULTIBAND model, which could generate different progression bandwidths for different road sections [7]. Subsequently, Stamatiadis and Gartner extended the MULTIBAND model as a solution for network coordination by developing the MULTIBAND-96 model [8]. Gartner and Stamatiadis, thereafter, proposed an optimized solution algorithm specific to MULTIBAND-96 and, thereby, significantly improved the computational efficiency $[9,10]$. Concurrently, Chaudhary and Messer developed an arterial traffic signal coordination software-PASSER IV, which could optimize the progression bandwidth in grid networks [11]. In recent years, relevant scholars have further studied bandwidth-based methods and obtained relatively detailed results based on the MAXBAND or MULTIBAND model. Tian and Urbanik proposed a bandwidth-oriented signal coordination method based on the system partition technology, which divided a large signalized arterial into several subsystems and optimized each subsystem to obtain the maximum progression bandwidth [12]. Lu et al. noted that the MAXBAND model failed to consider the dispersion feature of a platoon and added the platoon dispersion model as a constraint to the MAXBAND model, thereby proposing a modified MAXBAND model [13]. Tian et al. conducted quantitative evaluations of two signal timing issues (phase sequence and number of intersections) related to the progression bandwidth. The research results indicated that the lead-lag phase more easily yielded the maximum progression bandwidth solutions compared with the leading and lagging phases. However, a larger number of intersections imply more difficulty in obtaining the maximum progression bandwidth [14]. Chen et al. found that the queue clearance time changed dynamically with the offset instead of being a fixed value and accordingly derived the functional relation between the two parameters. Next, this functional relation was combined with MAXBAND to develop an improved MAXBAND model considering the dynamic queue clearance time [15]. Lin et al. proposed a new mixed-integer nonlinear programming model with the aim of providing maximum nonstops [16]. In consideration of the issue that the MAXBAND and MULTIBAND models may not be able to provide maximum progression bandwidths under certain weight factors, Lu et al. proposed a new type of maximum progression bandwidth model suitable for nonbalanced bandwidth demands by introducing proportional impact factors [17]. The overlapping and split phases are two classical phase design methods; however, the MAXBAND considers only the overlapping phase. To overcome this limitation, Lu et al. developed a bandwidth-oriented model with a comprehensive consideration of both the overlapping and split phases [18]. Cesme and Furth presented an inductive progression coordinated control method based on self-organizing traffic signals using secondary extension and dynamic coordination [19]. Li proposed a two-phase approach to solve the problem of travel time uncertainty. In this approach, first, the modified MAXBAND model was used to generate multiple optimal or suboptimal plans; next the Monte Carlo method was applied to simulate random travel time, evaluate the plans, and rank them according to their reliability [20]. Gomes introduced a vehicle arrival function into the signal coordination control and then developed a new bandwidth-oriented model. And then, he provided a detailed analysis of the pulse arrival and Gaussian arrival [21]. Ye et al. noted that the available coordination control method could not accurately calculate the queue clearance length and, therefore, developed a calculation formula specific to it that was incorporated into the coordination control model [22]. Based on the MAXBAND model, Yang et al. proposed a multipath maximum progression bandwidth model which could provide progression bands for multiple paths on an arterial [23]. Zhang et al. considered the constraint of the MULTIBAND model requiring the progression line to be symmetrical and proposed an asymmetric multiband model allowing the progression line to be asymmetrical-the AM-Band model [24]. Kim et al. proposed a dynamic progression bandwidth analysis method to adjust the bandwidth dynamically using closed loop signal data [25]. Based on the MAXBAND model, Dai et al. developed a new bandwidth-based model suitable for a bus lane system by taking into consideration factors such as the bus speed, location of the bus stops, and dwell time [26]. The existing models are highly dependent on fixed green splits; to overcome this shortcoming, Shirvani and Maleki proposed a modified multibandwidth model based on the acceptance degree of a bandwidth by using fuzzy control theory [27]. Zhang et al. studied long arterials and grid networks and then developed two bandwidth-oriented models (denoted as MAXBANDLA and MAXBANDGN models) to address the signal coordination problems of long arterials and grid networks [28]. When a common signal cycle is applied to a minor intersection, whose actual signal cycle length is approximately half of the common signal cycle length; it tends to cause an excessive delay for drivers on a crossing road. Therefore, Zhou et al. proposed an uneven double cycle control method to solve the above issue [29]. By introducing concepts such as the green centre line, ideal intersection position, and ideal intersection spacing, Lu et al. proposed a network progression band model with the minimum time difference between the actual and ideal green centre point as the objective function [30].

In general, all the bandwidth-based models mentioned above considered the through traffic flows as their objects and assigned the progression bands accordingly. On an arterial, the through traffic flows generally have larger volumes and should be awarded a higher priority and consequently assigned the progression bands. However, at certain times or nodes, when the left-turn traffic flows have a higher 


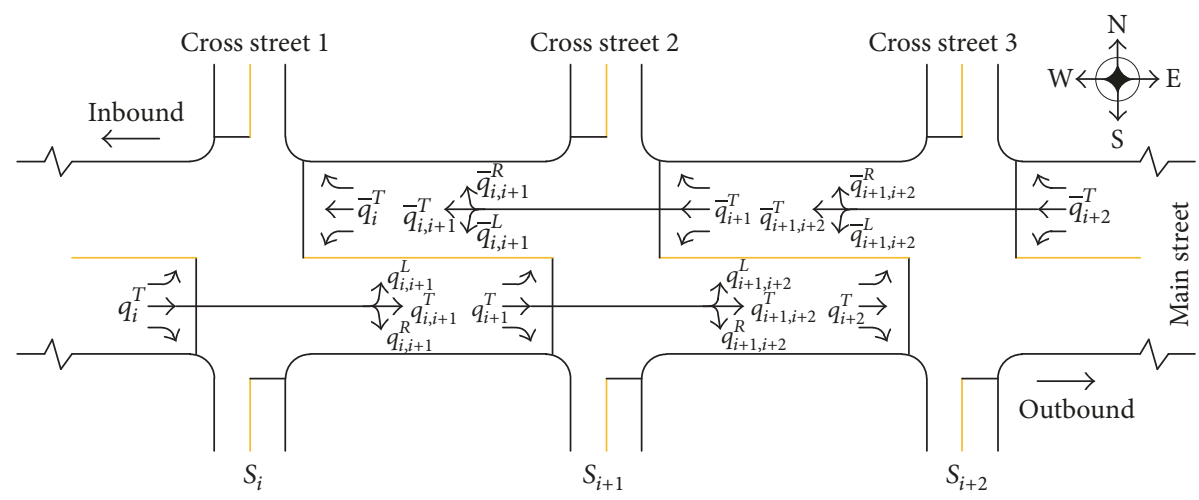

Figure 1: Comparison of the coordinated traffic flows.

volume than the through traffic flows, it would be inappropriate to continue to provide the progression bands to the through traffic flows. Under such conditions, it is obvious that converting the progression bands to the left-turn traffic flows, which have a higher priority then, could result in better traffic control. An arterial consisting of three signalized intersections, as shown in Figure 1, was selected as an example to illustrate the focus issue of this paper.

$q_{i}^{T}$ denotes the outbound through traffic flows at $S_{i}$ (west approach); $q_{i}^{T}$, driving from $S_{i}$ to $S_{i+1}$, will divide into three streams at $S_{i+1}$, namely, left-turn traffic flow $q_{i, i+1}^{L}$, through traffic flow $q_{i, i+1}^{T}$, and right-turn traffic flow $q_{i, i+1}^{R} ; \bar{q}_{i+2}^{T}$ denotes the inbound through traffic flows at $S_{i+2}$ (east approach), and similar to $q_{i}^{T}, \bar{q}_{i+2}^{T}$ will also divide into three streams at $S_{i+1}$ when driving from $S_{i+2}$ to $S_{i+1}$, namely, left-turn traffic flow $\bar{q}_{i+1, i+2}^{L}$, through traffic flow $\bar{q}_{i+1, i+2}^{T}$, and right-turn traffic flow $\bar{q}_{i+1, i+2}^{R}$. The other symbols follow the same explanation.

The available bandwidth-based methods typically consider the arterial through traffic flows (such as outbound through traffic flows $q_{i, i+1}^{T}$ and $q_{i+1, i+2}^{T}$, inbound through traffic flows $\bar{q}_{i+1, i+2}^{T}$ and $\bar{q}_{i, i+1}^{T}$ ) as their coordination subjects and assign progression bands accordingly. By this approach, the through traffic flows can pass through the entire arterial without any stops or with only a few stops, thereby achieving a good passing efficiency. Nevertheless, when the left-turn traffic flows at $S_{i+1}\left(q_{i, i+1}^{L}\right.$ and $\left.\bar{q}_{i+1, i+2}^{L}\right)$ have higher volumes than the through traffic flows $\left(q_{i, i+1}^{T}\right.$ and $\left.\bar{q}_{i+1, i+2}^{T}\right)$, and consequently have a higher priority and more urgent demand of progression band, it would be inappropriate to still coordinate the through traffic flows; instead the progression bands should be relayed to the left-turn traffic flows to achieve a better passing efficiency.

Summarizing, the issue to be examined in this study is as follows: first, we assume that the bidirectional left-turn traffic flows $\left(q_{i, i+1}^{L}\right.$ and $\left.\bar{q}_{i+1, i+2}^{L}\right)$ have a higher demand for the progression bands than the through traffic flows $\left(q_{i, i+1}^{T}\right.$ and $\left.\bar{q}_{i+1, i+2}^{T}\right)$ at $S_{i+1}$. Therefore, the bidirectional left-turn traffic flows $\left(q_{i, i+1}^{L}\right.$ and $\left.\bar{q}_{i+1, i+2}^{L}\right)$ will be taken into account as the objects. In this work, they will first be considered for modelling, which is significantly different from the existing methods. Next, outbound through traffic flows $q_{i+1, i+2}^{T}$ from
$S_{i+1}$ to $S_{i+2}$ and inbound through traffic flow $\bar{q}_{i, i+1}^{T}$ from $S_{i+1}$ to $S_{i}$ will also be considered during the modelling. This suggests that it is possible to concurrently synchronize the left-turn and through traffic flows.

In addition, we note that the number of upstream and downstream intersections is equal at $S_{i+1}$ in Figure 1. However, this is not necessary, that is, it is not required that $S_{i+1}$ be located in the middle of the arterial road. Figure 1 is only used to better illustrate the issue described in this paper.

The remainder of this paper is organized as follows. In Section 2, a general maximum progression model is presented, which can simultaneously coordinate the leftturn and through traffic flows and handle all the patterns of the left-turn phases by introducing two binary variables. In Section 3, a case study is discussed to compare the proposed model with the MULTIBAND model by using the traffic simulation software, VISSIM. Section 4 provides the conclusions with the final remarks and a description of possible future work.

\section{Model Formulations}

The necessity of coordinating the bidirectional left-turn traffic flows at $S_{i+1}$ was described in Section 1. There are four possible patterns of left-turn phases at $S_{i+1}$, as shown in Figure 2.

Figure 2 shows the four possible patterns of the left-turn phases. However, some readers may question the nonconsideration of the symmetric phase (the outbound and inbound green times for the through flows are equal; outbound and inbound green times for the left-turn flows are also equal) at $S_{i+1}$ in Figure 2. In fact, the symmetric phase is only a special case of Patterns I and II, and the specific explanation is as follows. From Figure 2(a), we can see that when the time of phase (B) in Pattern I is zero, Pattern I becomes Pattern VI, as shown in Figure 3(b). Similarly, we can see from Figure 2(b) that when the time of phase (B) in Pattern II is zero, Pattern II becomes Pattern V, as shown in Figure 3(a). Patterns V and VI are two possible patterns of the symmetric phase.

2.1. General Model of Simultaneously Optimizing Offsets and Phase Sequences. According to the above analysis, there are various patterns of left-turn phases at $S_{i+1}$ (see Figures 2 and $3)$. In this section, a general maximum progression model 
Pattern I: outbound left leads/inbound leads

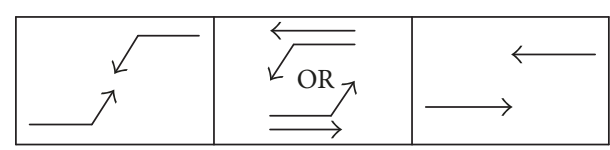

(A)

(B)

(C)

(a)

Pattern III: outbound left leads/inbound lags

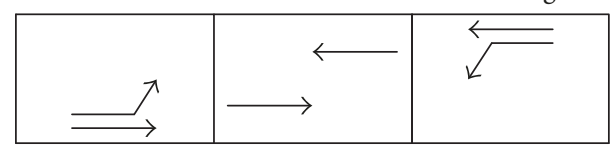

(A)
(B)

(c)
Pattern II: outbound left lags/inbound lags

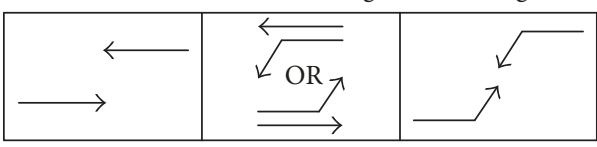

(A)

(B)

(C)

(b)

Pattern IV: outbound left lags/inbound leads

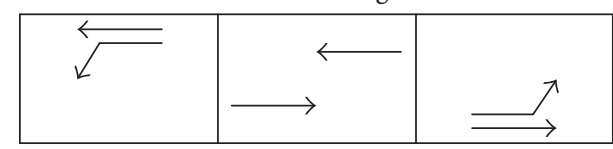

(A)

(B)

(C)

(d)

FIgURE 2: Four possible patterns of the left-turn phases (Gartner et al. [7]).

Pattern V: outbound and inbound left lags/through leads

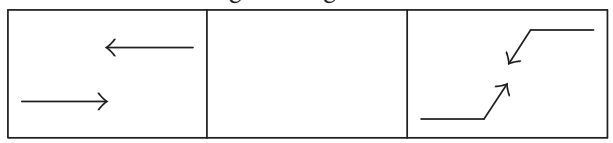

(A)
(B)

(a)
Pattern VI: outbound and inbound left leads/through lags

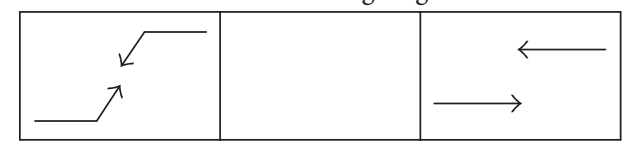

(A)

(C)

(b)

FIgure 3: Two possible patterns of the symmetric phase.

that can deal with all the above patterns of the left-turn phases is established by introducing two new binary variables $\delta_{i+1}$ and $\bar{\delta}_{i+1}$. In addition, key notations involved during the modelling are denoted as per the list in Notations.

To avoid repetition, we select Pattern I (see Figure 2) as the example for modelling; the modelling processes for other patterns can be conducted similarly. The time-space diagram for Pattern I based on the MULTIBAND model (Gartner et al. [7]) is presented in Figure 4.

Owing to the differences in the modelling objects (through traffic flows are taken as the object in MULTIBAND model, whereas left-turn and through traffic flows are simultaneously considered as the objects in the general model), the loop integer and inequality constraints in the MULTIBAND model cannot be directly applied to the general model. Thus, the new loop integer and inequality constraints suitable for the general model established in this paper are deduced as follows.

First, the derivation process of the new loop integer constraints is defined here. $\phi_{i, i+1}$ and $\bar{\phi}_{i, i+1}$ in Figure 4 can be expressed as

$$
\begin{aligned}
& \phi_{i, i+1}=0.5 r_{T i}+w_{i}+t_{i, i+1}-\tau_{i+1}-w_{i, i+1}-0.5 r_{i+1}, \\
& \bar{\phi}_{i, i+1}=0.5 \bar{r}_{T i}+\bar{w}_{i}-\bar{\tau}_{i}+\bar{t}_{i, i+1}-\bar{w}_{i+1}-0.5 r_{i+1} .
\end{aligned}
$$

In Figure 4, (3) is applicable from Point $A$ to $B$.

$$
\bar{\phi}_{i, i+1}+\phi_{i, i+1}+\Delta_{i}=n_{i, i+1} .
$$

Combining (1)-(3), the new loop integer constraint between $S_{i}$ and $S_{i+1}$ can be expressed by

$$
\begin{aligned}
& \left(0.5 r_{T i}+0.5 \bar{r}_{T i}-r_{i+1}-0.5 g_{L i}+0.5 \bar{g}_{L i}\right)+\left(w_{i}+\bar{w}_{i}\right) \\
& \quad-\left(w_{i, i+1}+\bar{w}_{i+1}\right)+\left(t_{i, i+1}+\bar{t}_{i, i+1}\right) \\
& \quad+\left(\delta_{i} g_{L i}-\bar{\delta}_{i} \bar{g}_{L i}\right)-\left(\bar{\tau}_{i}+\tau_{i+1}\right)=n_{i, i+1} .
\end{aligned}
$$
by

Similarly, $\phi_{i+1, i+2}$ and $\bar{\phi}_{i+1, i+2}$ in Figure 4 can be expressed

$$
\begin{aligned}
\phi_{i+1, i+2}= & 0.5 r_{i+1}+w_{i+1}+t_{i+1, i+2}-\tau_{i+2}-w_{i+2} \\
& -0.5 r_{T i+2}, \\
\bar{\phi}_{i+1, i+2}= & 0.5 r_{i+1}+\bar{w}_{i+2, i+1}-\bar{\tau}_{i+1}+\bar{t}_{i+1, i+2}-\bar{w}_{i+2} \\
& -0.5 \bar{r}_{T i+2} .
\end{aligned}
$$

In Figure 4, (7) is applicable from Point $C$ to $D$.

$$
\bar{\phi}_{i+1, i+2}+\phi_{i+1, i+2}-\Delta_{i+2}=n_{i+1, i+2} \text {. }
$$

Combining (5)-(7), the new loop integer constraint between $S_{i+1}$ and $S_{i+2}$ can be expressed by

$$
\begin{aligned}
\left(r_{i+1}\right. & \left.-0.5 r_{T i+2}-0.5 \bar{r}_{T i+2}+0.5 g_{L i+2}-0.5 \bar{g}_{L i+2}\right) \\
& +\left(w_{i+1}+\bar{w}_{i+2, i+1}\right)-\left(w_{i+2}+\bar{w}_{i+2}\right) \\
& +\left(t_{i+1, i+2}+\bar{t}_{i+1, i+2}\right)-\left(\delta_{i+2} g_{L i+2}-\bar{\delta}_{i+2} \bar{g}_{L i+2}\right) \\
& -\left(\tau_{i+2}+\bar{\tau}_{i+1}\right)=n_{i+1, i+2} .
\end{aligned}
$$




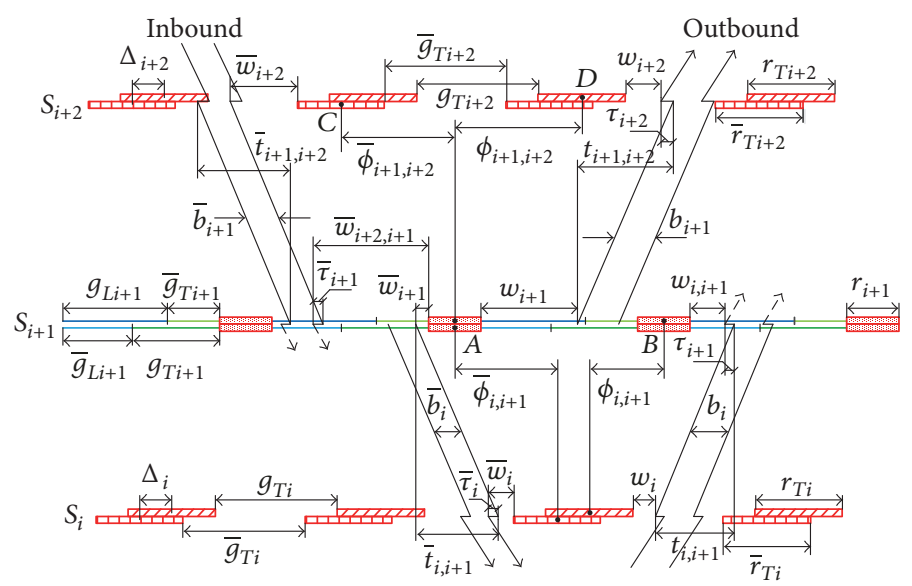

FIgURE 4: Time-space diagram for Pattern I.

Second, because the new inequality constraints are relatively simple and can be obtained directly from the time-space diagram; the corresponding expressions are directly provided in this section (see constraints represented in (15)-(18)).

$S_{i}, S_{i+1}$, and $S_{i+2}$ are considered as the model subjects, and similar to the MULTIBAND model, the objective is to maximize the sum of the two-way weight progression bandwidths. The general model established in this paper can be expressed as follows.

$$
\begin{array}{ll}
\max & \sum_{j=i}^{i+1}\left(\alpha_{j} b_{j}+\bar{\alpha}_{j} \bar{b}_{j}\right) \\
\text { s.t. } \quad & (4),(8) \\
& \left(1-k_{i}\right) \bar{b}_{i} \geq\left(1-k_{i}\right) k_{i} b_{i}, \quad i=1, \ldots, n-1 \\
& \frac{1}{C_{2}} \leq z \leq \frac{1}{C_{1}} \\
& \left(\frac{d_{i}}{f_{i}}\right) z \leq t_{i, i+1} \leq\left(\frac{d_{i}}{e_{i}}\right) z \\
& \left(\frac{\bar{d}_{i}}{\bar{f}_{i}}\right) z \leq \bar{t}_{i, i+1} \leq\left(\frac{\bar{d}_{i}}{\bar{e}_{i}}\right) z \quad i=1, \ldots, n-1 \\
& \left(\frac{d_{i}}{h_{i}}\right) z \leq\left(\frac{d_{i}}{d_{i+1}}\right) t_{i+1, i+2}-t_{i, i+1} \leq\left(\frac{d_{i}}{\bar{g}_{i}}\right) z \\
& \left(\frac{\bar{d}_{i}}{\bar{h}_{i}}\right) z \leq\left(\frac{\bar{d}_{i}}{\bar{d}_{i+1}}\right) \bar{t}_{i+1, i+2}-\bar{t}_{i, i+1} \leq\left(\frac{\bar{d}_{i}}{\bar{g}_{i}}\right) z \\
& w_{j} \geq 0 \quad j=i, i+2 \quad i=1, \ldots, n-2 \\
\bar{w}_{j} \geq 0 \quad j=i, i+2 &
\end{array}
$$

$$
\begin{aligned}
& w_{j, j+1}-\delta_{j+1}\left(1-\bar{r}_{T j+1}\right) \geq 0 \\
& w_{j+1}-\bar{\delta}_{j+1}\left(1-\bar{r}_{L j+1}\right) \geq 0 \\
& \bar{w}_{j+2, j+1}-\bar{\delta}_{j+1}\left(1-r_{T j+1}\right) \geq 0 \\
& \bar{w}_{j+1}-\delta_{j+1}\left(1-r_{L j+1}\right) \geq 0 \\
& w_{j}+b_{j} \leq 1-r_{T j} \quad j=i \\
& \bar{w}_{j}+\bar{b}_{j} \leq 1-\bar{r}_{T j}+\bar{\tau}_{j} \\
& w_{j+2}+b_{j+1} \leq 1-r_{T j+2}-\tau_{j+2} \\
& \bar{w}_{j+2}+\bar{b}_{j+1} \leq 1-\bar{r}_{T j+2} \\
& w_{j, j+1}+b_{j} \leq 1-r_{L j+1}+\delta_{j+1}\left(1-\bar{r}_{T j+1}\right)-\tau_{j+1} \\
& w_{j+1}+b_{j+1} \leq 1-r_{T j+1}+\bar{\delta}_{j+1}\left(1-\bar{r}_{L j+1}\right) \\
& \bar{w}_{j+2, j+1}+\bar{b}_{j+1} \\
& \leq 1-\bar{r}_{L j+1}+\bar{\delta}_{j+1}\left(1-r_{T j+1}\right)+\bar{\tau}_{j+1} \\
& \bar{w}_{j+1}+\bar{b}_{j} \leq 1-\bar{r}_{T j+1}+\delta_{j+1}\left(1-r_{L j+1}\right) \\
& z, b_{j}, \bar{b}_{j} \geq 0 \quad j=i, i+1 \\
& n_{j, j+1}, n_{j+1, j+2} \quad \text { integers } j=i \\
& \delta_{j} \bar{\delta}_{j} \quad \text { binary integers } j=i, i+1, i+2 . \\
& w^{\prime}
\end{aligned}
$$

In the proposed model, the objective function and constraints are linear. Particularly, because the variables in 
TABLE 1: Correspondence between the values of $\delta_{i+1}$ and $\bar{\delta}_{i+1}$ and the phase patterns.

\begin{tabular}{lccc}
\hline Pattern & $\delta_{i+1}$ & $\bar{\delta}_{i+1}$ & Note \\
\hline I or VI & 0 & 1 & Outbound left leads/inbound leads or outbound and inbound left leads/through lags \\
II or V & 1 & 0 & Outbound left lags/inbound lags or outbound and inbound left lags/through leads \\
III & 0 & 0 & Outbound left leads/inbound lags \\
IV & 1 & 1 & Outbound left lags/inbound leads \\
\hline
\end{tabular}

constraints (20) and (21) are integers, the proposed model is a mixed-integer linear programming model. In the above formulations, the decision variables include the bandwidth $\left[b_{j}, \bar{b}_{j}(j=i, i+1)\right]$, reciprocal of the cycle length $[z]$, inference variables $\left[w_{j}, \bar{w}_{j}(j=i, i+1, i+2) ; w_{j, j+1}, \bar{w}_{j+2, j+1}(j=\right.$ $i)$, travel time $\left[t_{j, j+1}, \bar{t}_{j, j+1}(j=i, i+1)\right]$, and integer variables $\left[\delta_{j}, \bar{\delta}_{j}(j=i, i+1, i+2) ; n_{j, j+1}(j=i, i+1)\right]$. The parameters include the weighting coefficient $\left[\alpha_{j}, \bar{\alpha}_{j}(j=\right.$ $i, i+1)]$, target ratio of the bandwidth $\left[k_{j}(j=i, i+1)\right]$, boundaries of the cycle length $\left[C_{1}, C_{2}\right]$, distance between the intersections $\left[d_{j}, \bar{d}_{j}(j=i, i+1)\right]$, boundaries of the travel speed $\left[e_{j}, f_{j}, \bar{e}_{j}, \bar{f}_{j}(j=i, i+1)\right]$, boundaries of the travel speed variation $\left[g_{j}, h_{j}, \bar{g}_{j}, \bar{h}_{j}(j=i)\right]$, the red time $\left[r_{T j}, \bar{r}_{T j}(j=i, i+1, i+2) ; r_{j+1}, r_{L j+1}, \bar{r}_{L j+1}(j=i)\right]$, the green time $\left[g_{L j}, \bar{g}_{L j}(j=i, i+2)\right]$, and the initial queue clearance time $\left[\tau_{j}, \bar{\tau}_{j}(j=i, i+1, i+2)\right]$.

Function (9) is the objective function of the proposed model that aims to maximize the left-turning and through progression bandwidths. Constraint (11) is used to indicate the direction with the higher weight and identify the outbound and inbound directions. The direction with the higher weight will be assigned the larger progression bandwidth. Constraint (12) is used to limit the value range of the cycle length. Constraint (13) is employed to limit the travel speed on the road section to within a reasonable range. Constraint (14) is applied to limit the travel speed change between consecutive road sections to within a reasonable range. Constraint (15) is used to limit the minimum values of $w_{j}$ and $\bar{w}_{j}(j=i, i+2)$ at $S_{i}$ and $S_{i+2}$. Constraint (16) is used to limit the minimum values of $w_{j, j+1}, w_{j+1}, \bar{w}_{j+2, j+1}$, and $\bar{w}_{j+1}$ $(j=i)$ at $S_{i+1}$. Constraint (17) limits the maximum values of $w_{j}, \bar{w}_{j}, w_{j+2}$, and $\bar{w}_{j+2}(j=i)$ at $S_{i}$ and $S_{i+2}$. Constraint (18) is applied to limit the maximum values of $w_{j, j+1}, w_{j+1}$, $\bar{w}_{j+2, j+1}$, and $\bar{w}_{j+1}(j=i)$ at $S_{i+1}$. Constraint (19) is used to indicate that the variables in this constraint are nonnegative. Constraint (20) specifies that the variables in this constraint are integers. Constraint (21) indicates that the variables in this constraint are valued as 0 or 1 .

2.2. Correspondence Relationships between Binary Variables and Phase Sequences. Although the derivation of the general model proposed in this paper is based on Pattern I, the general model can also deal with the remaining phase patterns (Patterns II to VI) by introducing two new binary variables $\left(\delta_{i+1}\right.$ and $\left.\bar{\delta}_{i+1}\right)$ into the inequality constraints (see constraints (16) and (18)). This implies that the maximum progression model established herein is a general mathematical model that can deal with all the above-mentioned phase patterns.
The correspondence between the values of $\delta_{i+1}$ and $\bar{\delta}_{i+1}$ and the phase patterns is shown in Table 1.

As can be seen from Table $1, \delta_{i+1}=0$ and $\bar{\delta}_{i+1}=$ 1 correspond to two patterns, that is, Patterns I and VI. A question to be addressed is how to distinguish between Patterns I and VI when the values $\delta_{i+1}=0$ and $\bar{\delta}_{i+1}=1$ are obtained after the general model is solved. The specific method to achieve this is given below. Before the model is solved, the green time of each traffic flow at $S_{i+1}$ is obtained (i.e., the green time is a known parameter). If the outbound and inbound green times for the through traffic flows are equal $\left(g_{T i+1}=\bar{g}_{T i+1}\right)$ and so are for the left-turn traffic flows $\left(g_{L i+1}=\bar{g}_{L i+1}\right)$, then the case corresponds to Pattern VI, otherwise it corresponds to Pattern I. Similarly, when $\delta_{i+1}=1$ and $\bar{\delta}_{i+1}=0$ (as listed in Table 1), Patterns II and V can be distinguished.

\section{Numerical Example}

3.1. Basic Parameters of the Arterial. Yinhai road including five signalized intersections in the Yiwu city is selected to verify its validity of the established general model. The distribution of each intersection on the arterial is shown in Figure 5. Chouzhou road is an important traffic attraction because various trade marts are located on this road, which cause more traffic flows to turn left at $S_{3}$. Therefore, Yinhai road provides a good testing arterial to verify the effectiveness of the model proposed herein. In Table 2, LT, TH, and RT are the abbreviations for left-turn, through, and right-turn, respectively; and $\mathrm{W}, \mathrm{N}, \mathrm{E}$, and $\mathrm{S}$ denote the west, north, east, and south approach of the intersections, respectively. The design speed for the progression bands in both the outbound and inbound directions on the arterial is $45 \mathrm{~km} / \mathrm{h}$.

3.2. Time-Space Diagrams. Based on the traffic flow data presented in Table 2 for each intersection and the Webster theory, the distribution ratio of the phase green time for each intersection is listed in Table 3 (saturation flow of one lane is assumed as $1800 \mathrm{veh} / \mathrm{h})$.

Table 2 shows that, at $S_{3}$, the outbound left-turn traffic volume $(1310 \mathrm{veh} / \mathrm{h})$ is larger than the outbound through traffic volume $(828 \mathrm{veh} / \mathrm{h})$, and the inbound left-turn traffic volume $(932 \mathrm{veh} / \mathrm{h})$ is also larger than the inbound through traffic volume $(546 \mathrm{veh} / \mathrm{h})$. Thus, the left-turn traffic flows at $S_{3}$ have a stronger and more urgent demand for the progression band than the through traffic flows. An attempt has been also made to assign the progression bandwidths to the outbound and inbound left-turn traffic flows at $S_{3}$.

The method is to input the distances between the intersections, design speed of the progression bands, signal timing 
TABLE 2: Traffic flow volumes at each intersection on the arterial (veh/h).

\begin{tabular}{|c|c|c|c|c|c|c|c|c|c|c|c|c|c|c|c|}
\hline \multirow{2}{*}{ Approach } & \multicolumn{3}{|c|}{$S_{1}$} & \multicolumn{3}{|c|}{$S_{2}$} & \multicolumn{3}{|c|}{$S_{3}$} & \multicolumn{3}{|c|}{$S_{4}$} & \multicolumn{3}{|c|}{$S_{5}$} \\
\hline & LT & $\mathrm{TH}$ & RT & LT & TH & RT & LT & $\mathrm{TH}$ & RT & LT & $\mathrm{TH}$ & RT & LT & $\mathrm{TH}$ & RT \\
\hline $\mathrm{W}$ & 902 & 1380 & 190 & 884 & 1354 & 130 & 1310 & 828 & 100 & 586 & 1040 & 96 & 610 & 1100 & 220 \\
\hline $\mathrm{N}$ & 514 & 400 & 210 & 442 & 468 & 220 & 440 & 711 & 300 & 500 & 870 & 174 & 448 & 690 & 186 \\
\hline E & 478 & 746 & 120 & 504 & 684 & 100 & 932 & 546 & 258 & 600 & 1072 & 216 & 560 & 1250 & 224 \\
\hline$S$ & 510 & 394 & 474 & 440 & 472 & 442 & 442 & 705 & 454 & 490 & 900 & 390 & 452 & 681 & 320 \\
\hline
\end{tabular}

TABLE 3: Distribution ratio of phase green time for each intersection.

\begin{tabular}{|c|c|c|c|c|c|c|c|c|}
\hline \multirow{2}{*}{ Intersection } & \multicolumn{2}{|c|}{$\mathrm{W}$} & \multicolumn{2}{|c|}{$\mathrm{N}$} & \multicolumn{2}{|c|}{$\mathrm{E}$} & \multicolumn{2}{|c|}{$S$} \\
\hline & LT & $\mathrm{TH}$ & LT & $\mathrm{TH}$ & LT & $\mathrm{TH}$ & LT & $\mathrm{TH}$ \\
\hline$S_{1}$ & 0.36686 & 0.49784 & 0.18542 & 0.14430 & 0.17244 & 0.30342 & 0.18542 & 0.14430 \\
\hline$S_{2}$ & 0.37789 & 0.48846 & 0.15945 & 0.17027 & 0.18182 & 0.29239 & 0.15945 & 0.17027 \\
\hline$S_{3}$ & 0.47258 & 0.31499 & 0.15945 & 0.17100 & 0.35456 & 0.19697 & 0.15945 & 0.17100 \\
\hline$S_{4}$ & 0.21645 & 0.38672 & 0.18038 & 0.21645 & 0.21645 & 0.38672 & 0.18038 & 0.21645 \\
\hline$S_{5}$ & 0.22006 & 0.44464 & 0.16306 & 0.16594 & 0.22636 & 0.45094 & 0.16306 & 0.16594 \\
\hline
\end{tabular}

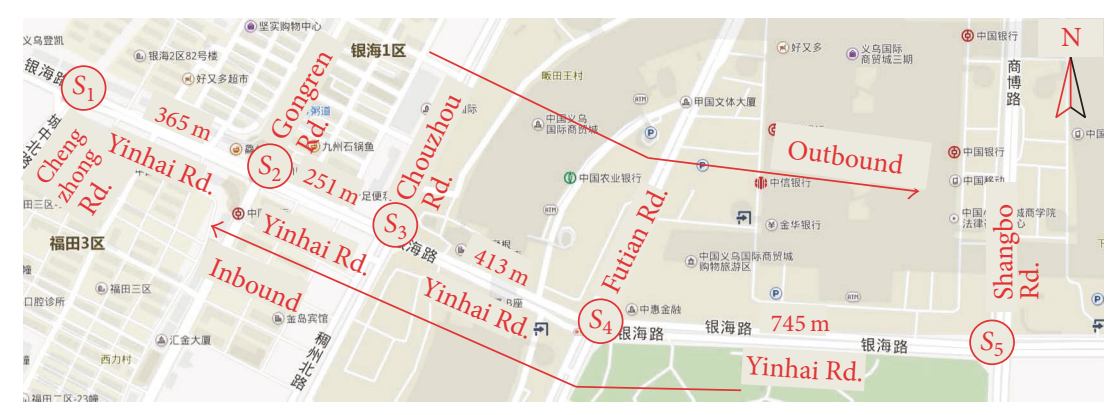

Figure 5: Distribution of the intersections on the arterial.

(as shown in Table 3), common cycle length (calculated as $100 \mathrm{~s}$ using the Webster cycle formula, assuming a total lost time at each intersection is $12 \mathrm{~s}$ and the traffic flow volume data as presented in Table 2), and other data into the MULTIBAND model and established general model. These models are solved by using the optimization tool, LINGO. The solution of the general model is $\delta_{3}=0$ and $\bar{\delta}_{3}=1$, exhibiting that the optimal pattern at $S_{3}$ is Pattern I or Pattern V (see Table 1). Simultaneously, it can be seen from Table 3 that at $S_{3}$ the outbound distribution ratio of the green time (0.31499) for the through flows is not equal to that of the inbound one $(0.19697)$ for the through flows. In addition, the outbound distribution ratio of the green time (0.47258) for the left-turn traffic flows is also not equal to the inbound ratio $(0.35456)$ for the left-turn flows. Therefore, the optimal phase pattern obtained by the general model at $S_{3}$ is Pattern I. The solution of the MULTIBAND model is $\delta_{3}=1$ and $\bar{\delta}_{3}=1$, indicating that the optimal pattern obtained by the MULTIBAND model at $S_{3}$ is Pattern II (the correspondence between $\delta_{i}$ and $\bar{\delta}_{i}$ and phase patterns is shown in [7]). The optimal solutions obtained by LINGO are displayed in the time-space diagrams, as displayed in Figure 6 (for the MULTIBAND model) and Figure 7 (for the general model established in this paper), respectively.

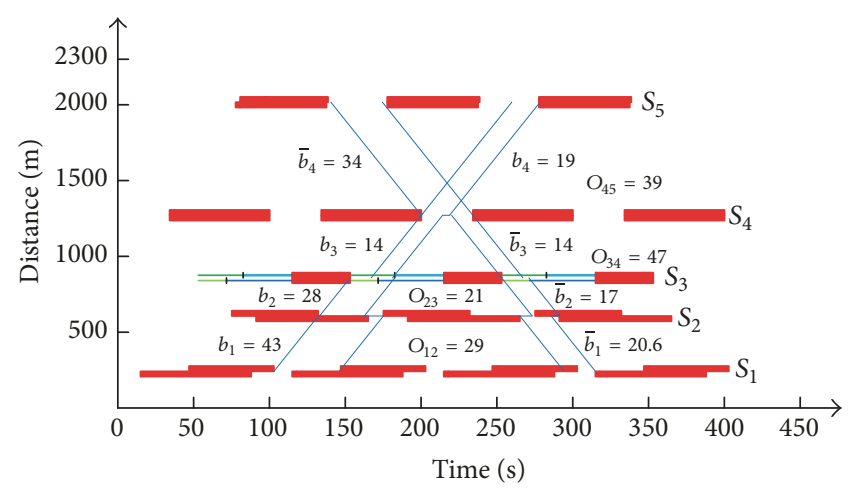

FIgURE 6: Time-space diagram generated by the MULTIBAND model.

From the above analysis, although the MULTIBAND model has optimal phase patterns that are different from those of the established general model, both models yielded the theoretical maximum progression bandwidth corresponding to the optimal phase pattern. Because their modelling objects are different, the derived progression bands still have the following differences: 


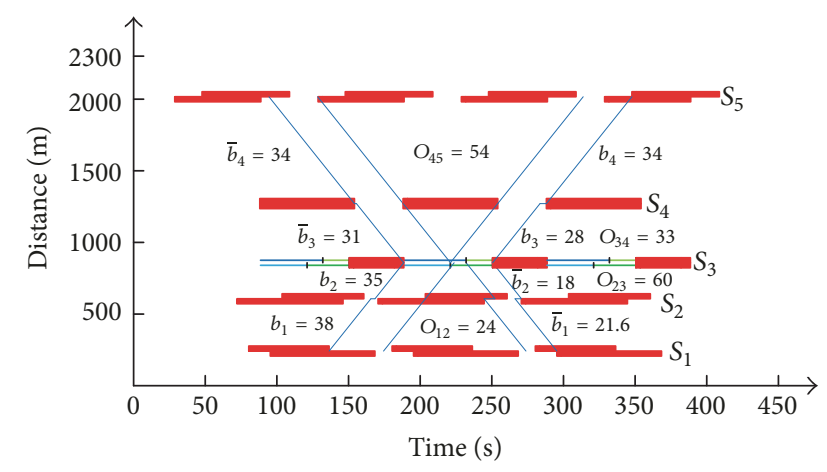

FIgURE 7: Time-space diagram generated by the proposed general model.

In Figure 6, at $S_{3}$, the MULTIBAND model selects the outbound and inbound through traffic flows as its modelling subjects to provide the progression bands; the bandwidth for the outbound through traffic flow between $S_{2}$ and $S_{3}$ is $b_{2}=28$ and that for the inbound through traffic flow between $S_{3}$ and $S_{4}$ is $\bar{b}_{3}=14$. In Figure 7, the general model considers the outbound and inbound left-turn traffic flows as its modelling subjects to provide the progression bands; the bandwidth for the outbound left-turn traffic flow between $S_{2}$ and $S_{3}$ is $b_{2}=35$, and the bandwidth for the inbound leftturn traffic flow between $S_{3}$ and $S_{4}$ is $\bar{b}_{3}=31$. It can be seen that the two models differ from each other mainly in terms of the different modelling objects, and, thus, different progression bandwidths. At $S_{3}$, the MULTIBAND model provides a progression bandwidth of $b_{2}+\bar{b}_{3}=42$ (shown in Figure 6) for the through traffic flows, whereas the general model provides $b_{2}+\bar{b}_{3}=66$ (shown in Figure 7) for the left-turn traffic flows. The general model here, being superior to the MULTIBAND model in terms of the progression bandwidth, may be able to generate a better traffic control than the MULTIBAND model (such as less delay, stops, and travel time), which will be validated hereunder using traffic simulation.

3.3. Simulation and Results Analysis. To evaluate the proposed general model and MULTIBAND model, the average delay time, average vehicle stops, and average travel time were used as measures of effectiveness $\left(\mathrm{MOE}_{\mathrm{S}}\right)$, which were obtained using the traffic simulation software, VISSIM. The traffic signal synchronizing solutions generated by the two models were input into VISSIM (including the cycle length, offset, phase, and phase sequence). To avoid the randomness caused by a one-time traffic simulation, ten random seeds were selected to form ten situations of traffic simulation. It is decided to evaluate and compare the performance of the two models on two levels.

Level 1: Considering that the main aspect that differentiates the general model from the MULTIBAND model is that it provides the progression bands to the outbound left-turn $\left(q_{2,3}^{L}\right.$ from $S_{2}$ to $\left.S_{3}\right)$ and inbound left-turn traffic flows $\left(\bar{q}_{3,4}^{L}\right.$ from $S_{4}$ to $\left.S_{3}\right)$ instead of the outbound through $\left(q_{2,3}^{T}\right.$ from $S_{2}$ to $\left.S_{3}\right)$ and inbound through traffic flows $\left(\bar{q}_{3,4}^{T}\right.$ from $S_{4}$ to $\left.S_{3}\right)$ as defined

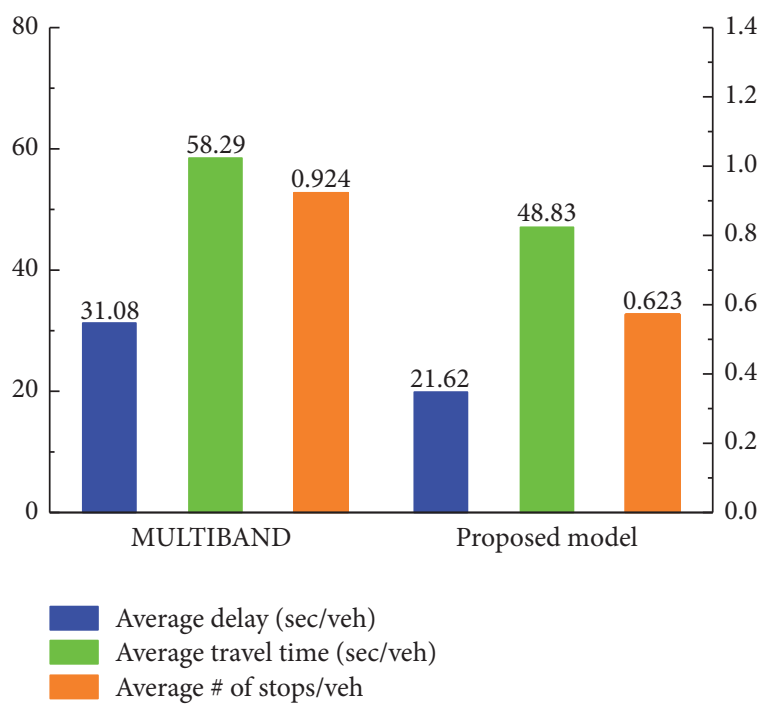

Figure 8: MOE comparison between the proposed general model and MULTIBAND model at Level 1.

by the MULTIBAND model. The traffic flows (including the left-turns and through traffic flows) between intersections $S_{2}$, $S_{3}$, and $S_{4}$ were selected as the evaluation objects for Level 1 to verify the effectiveness of coordinating left-turn flows. The traffic simulation data of the ten random seeds were averaged, and the obtained average delay time, average travel time, and average vehicle stops were used to compare the two models. See Figure 8 for the comparison results for Level 1 .

Figure 8 shows that, at Level 1, the average delay time, average travel time, and average vehicle stops obtained from the general model are smaller than those derived from the MULTIBAND model by $30.44 \%, 16.23 \%$, and $32.58 \%$, respectively, implying that at Level 1 it is feasible to provide the progression bands for the outbound and inbound leftturn traffic flows $\left(q_{2,3}^{L}\right.$ and $\left.\bar{q}_{3,4}^{L}\right)$ at $S_{3}$ and concurrently obtain a better traffic control. This validates the general model established in this paper.

Second, Level 2 considered the traffic flows in the entire arterial $\left(S_{1}, S_{2}, S_{3}, S_{4}\right.$, and $\left.S_{5}\right)$ as its evaluation objects. The traffic simulation data of the ten random seeds were also averaged, and the obtained average delay time, average travel time, and average vehicle stops were used to compare the two models. Refer to Figure 9 for the comparison results for Level 2.

Figure 9 shows that, at Level 2, the average delay time, average travel time, and average vehicle stops derived from the general model are smaller than those of the MULTIBAND model by $32.51 \%, 14.10 \%$, and $27.59 \%$, respectively. This implies that the general model established herein can obtain a better control than the MULTIBAND model in terms of the entire arterial.

Compared with Level 1 and Level 2 in the MULTIBAND model, the general model has a better control, as proved by its shorter average delay time, fewer average stops, and shorter average travel time. This validates the concept of providing 


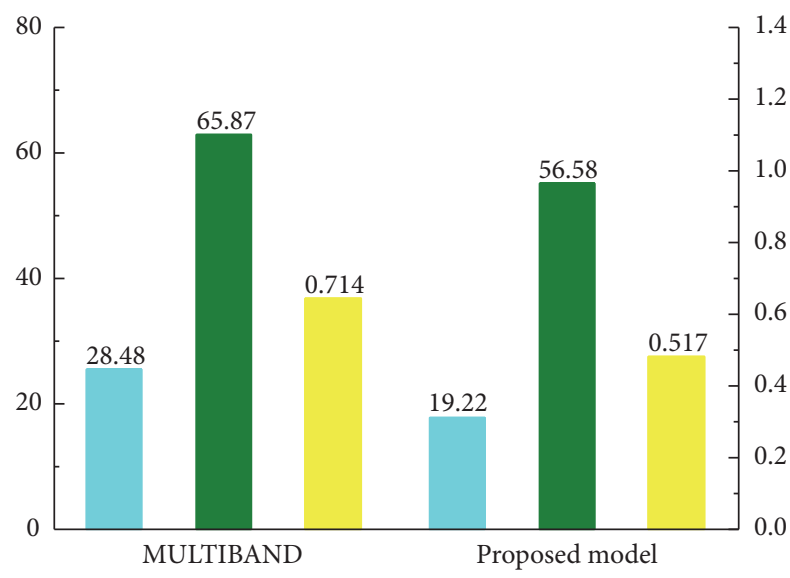

Average delay (sec/veh)
Average travel time (sec/veh)
Average \# of stops/veh

FIGURE 9: MOE comparison between the proposed general model and MULTIBAND model at Level 2.

the progression bands to the left-turn traffic flows to achieve a better control.

3.4. Discussion. It is necessary to highlight that the traffic flows on crossing roads were not considered in the traffic simulation evaluation, because of which the comprehensiveness of the performance evaluation based on the two models could be questioned. The reasons for why the traffic flows on the crossing roads were not included in the simulation evaluation are as follows: (1) Considering the traffic flow from the north approach at $S_{1}$ as an example, the traffic flow comes from the upstream intersection $\left(S_{\mathrm{N} 1}\right)$ on the north side of $S_{1}$. The delay time, vehicle stops, and travel time of this traffic flow are affected by the offset between $S_{\mathrm{N} 1}$ and $S_{1}$. Therefore, the simulation evaluation of such a traffic flow should be conducted on the north-south road from $S_{\mathrm{N} 1}$ to $S_{1}$ instead of the east-west arterial from $S_{1}$ to $S_{5}$; (2) all the bandwidth-based methods (MULTIBAND model and general model herein) consider the traffic flows on arterials as their research subjects; the traffic flows on the crossing roads intersecting the arterials should be evaluated on the corresponding crossing roads instead of on the arterial.

\section{Conclusions}

The existing bandwidth-based methods typically consider the through traffic flows on an arterial as their study object to provide the progression bands, implying that the through traffic flows have a higher priority and more urgent requirement for the same. However, when the left-turn traffic flows have a higher priority and more urgent demand for the progression bands than the through traffic flows, it would be inappropriate to still provide the progression bands to the through traffic flows. A better control may be achieved by selecting the left-turn traffic flows as the study object and providing them the progression bands. In this view, a general maximum progression model to concurrently synchronize the left-turn and through traffic flows was established in this paper by using time-space diagrams. The general model could deal with all the patterns of the left-turn phases by introducing two new binary variables. A numerical example was selected to compare the MULTIBAND model and general model established herein, the results of which indicated that the latter can effectively reduce the average delay time, average vehicle stops, and average travel time. Thus, the appropriateness and practicability of the general model established herein were validated.

In the future research work, the following two directions can be focused on: (1) the general model established herein considers two-way left-turn traffic flows (outbound left-turn traffic flow $q_{i, i+1}^{L}$ and inbound left-turn traffic flow $\bar{q}_{i+1, i+2}^{L}$ as shown in Figure 1) at $S_{i+1}$ as their study objects and provides progression bands accordingly. In the future, the general model could be expanded to include one-way leftturn traffic flows (such as outbound left-turn traffic flow $q_{i, i+1}^{L}$ ) and one-way through traffic flows (such as inbound through traffic flow $\bar{q}_{i+1, i+2}^{T}$ ) at $S_{i+1}$ as the study objects. (2) After the technology of the Internet of Vehicles is applied and matures in the future, the demand of the traffic flows on different roads in a road network for the progression bands could be obtained in real time and dynamically. Such traffic flows could then be selected as the study objects to assign the progression bands to better adapt to the traffic flow variation and provide the progression bands to traffic flows in urgent need more accurately.

\section{Notations}

$b_{i}\left(\bar{b}_{i}\right): \quad$ Outbound (inbound) progression

$\alpha_{i}\left(\bar{\alpha}_{i}\right): \quad$ Weight coefficient of outbound (inbound)

bandwidth $b_{i}\left(\bar{b}_{i}\right)$

$k_{i}$ : $\quad$ Target ratio between inbound bandwidth

$\bar{b}_{i}$ and outbound bandwidth $b_{i}$

$C_{1}, C_{2}$ : $\quad$ Upper limit and lower limit of cycle length

$z: \quad$ Reciprocal of cycle length

$r_{T i}\left(\bar{r}_{T i}\right): \quad$ Red time for through traffic flows in

outbound (inbound) direction at $S_{i}$

$g_{L i}\left(\bar{g}_{L i}\right): \quad$ Green time for left-turn traffic flows in outbound (inbound) direction at $S_{i}$

$t_{i, i+1}\left(\bar{t}_{i, i+1}\right):$ Travel time from $S_{i}\left(S_{i+1}\right)$ to $S_{i+1}\left(S_{i}\right)$ in outbound (inbound) direction

$\tau_{i}\left(\bar{\tau}_{i}\right): \quad$ Queue clearance time at $S_{i}$ in outbound (inbound) direction

$d_{i}\left(\bar{d}_{i}\right): \quad$ Distance between $S_{i}\left(S_{i+1}\right)$ and $S_{i+1}\left(S_{i}\right)$ in outbound (inbound) direction

$e_{i}, f_{i}\left(\bar{e}_{i}, \bar{f}_{i}\right)$ : Upper limit and lower limit of travel speed in outbound (inbound) direction

$g_{i}, h_{i}\left(\bar{g}_{i}, \bar{h}_{i}\right):$ Upper limit and lower limit of travel speed change in outbound (inbound) direction

$\delta_{i}\left(\bar{\delta}_{i}\right): \quad 0 / 1$ variables, depending on left-turn phase patterns

$\Delta_{i}: \quad$ Time difference between the centre of $r_{T i}$ to the nearest center of $\bar{r}_{T i} \Delta_{i}=\delta_{i} g_{L i}-0.5 g_{L i}-\bar{\delta}_{i} \bar{g}_{L i}+0.5 \bar{g}_{L i}$ 


$$
\begin{array}{ll}
w_{i}\left(\bar{w}_{i}\right): & \text { Time from the left-side (right-side) edge } \\
& \text { of progression band } b_{i}\left(\bar{b}_{i}\right) \text { at } S_{i} \text { to the } \\
& \text { right-side (left-side) edge of the adjacent } \\
& \text { red light in outbound (inbound) direction } \\
w_{i, i+1}\left(\bar{w}_{i+2, i+1}\right): & \text { Time from the left-side (right-side) edge } \\
& \text { of progression band } b_{i}\left(\bar{b}_{i+1}\right) \text { at } S_{i+1} \text { to the } \\
& \text { right-side (left-side) edge of the adjacent } \\
& \text { red light in outbound (inbound) direction } \\
& \text { Red time of the coordination direction at } \\
& S_{i+1} \text {, that is, green time on the cross road } \\
& \text { Time from the centre of an outbound } \\
& \text { (inbound) red lights at } S_{i} \text { to the center of } \\
& \text { the nearest outbound (inbound) red lights } \\
& \text { at } S_{i+1}: \\
& \text { The integer coefficient for loop integer } \\
& \text { constraint between } S_{i} \text { and } S_{i+1} \text {. }
\end{array}
$$

\section{Conflicts of Interest}

The authors declare that there are no conflicts of interest regarding the publication of this paper.

\section{Acknowledgments}

This research was supported by the National Natural Science Foundation of China (Grant no. 61174184) and Science and Technology Planning Project of Guangdong Province, China (Grant nos. 2016A030305001 and 2014B090904059).

\section{References}

[1] J. T. Morgan and J. D. C. Little, "Synchronizing traffic signals for maximal bandwidth," Operations Research, vol. 12, no. 6, pp. 896-912, 1964.

[2] J. D. C. Little, "The synchronization of traffic signals by mixedintegerlinearprogramming," Operations Research, vol. 14, no. 4, pp. 568-594, 1966.

[3] C. J. Messer, R. H. Whitson, C. L. Dudek, and E. J. Romano, "A variable-sequence multiphase progression optimization program," Transportation Research Record, vol. 445, pp. 24-33, 1973.

[4] J. D. Little, M. D. Kelson, and N. H. Gartner, MAXBAND: A Versatile Program for Setting Signals on Arteries And Triangular Networks, Massachusetts Institute of Technology, Cambridge, 1981.

[5] E. C.-P. Chang, S. L. Cohen, C. Liu, N. A. Chaudhary, and C. Messer, "MAXBAND-86: program for optimizing left-turn phase sequence in multiarterial closed networks," Transportation Research Record, no. 1181, pp. 61-67, 1988.

[6] H.-S. Tsay and L.-T. Lin, "New algorithm for solving the maximum progression bandwidth," Transportation Research Record, no. 1194, pp. 15-30, 1988.

[7] N. H. Gartner, S. F. Assmann, F. Lasaga, and D. L. Hous, "MULTI-BAND-a variable-bandwidth arteries progression scheme," Transportation Research Record, vol. 1287, pp. 212-222, 1990.

[8] C. Stamatiadis and N. H. Gartner, "MULTIBAND-96: a program for variable-bandwidth progression optimization of multiarterial traffic networks," Transportation Research Record, vol. 1554, no. 1, pp. 9-17, 1996.
[9] N. H. Gartner and C. Stamatiadis, "Progression optimization featuring arterial- and route-based priority signal networks," Journal of Intelligent Transportation Systems: Technology, Planning, and Operations, vol. 8, no. 2, pp. 77-86, 2004.

[10] N. H. Gartner and C. Stamatiadis, "Arterial-based control of traffic flow in urban grid networks," Mathematical and Computer Modelling, vol. 35, no. 5-6, pp. 657-671, 2002.

[11] N. A. Chaudhary and C. J. Messer, "PASSER IV: a program for optimizing signal timing in grid networks," Transportation Research Record, no. 1421, pp. 82-93, 1993.

[12] Z. Tian and T. Urbanik, "System partition technique to improve signal coordination and traffic progression," Journal of Transportation Engineering, vol. 133, no. 2, pp. 119-128, 2007.

[13] S. F. Lu, X. M. Liu, and S. Q. Dai, "Revised MAXBAND model for bandwidth optimization of traffic flow dispersion," in Proceedings of the ISECS International Colloquium on Computing, Communication, Control, and Management, CCCM 2008, pp. 85-89, China, August 2008.

[14] Z. Tian, V. Mangal, and H. Liu, "Effectiveness of lead-lag phasing on progression bandwidth," Transportation Research Record, no. 2080, pp. 22-27, 2008.

[15] N. N. Chen, Z. C. He, and Z. Yu, "Revised MAXBAND model considered variable queue clearance time," Journal of Wuhan University of Technology (Transportation Science and Engineering), vol. 33, no. 5, pp. 843-847, 2009.

[16] L.-T. Lin, L.-W. C. Tung, and H.-C. Ku, "Synchronized signal control model for maximizing progression along an arterial," Journal of Transportation Engineering, vol. 136, no. 8, pp. 727735,2010

[17] K. Lu, X. Zeng, L. Li, and J. Xu, "Two-way bandwidth maximization model with proration impact factor for unbalanced bandwidth demands," Journal of Transportation Engineering, vol. 138, no. 5, pp. 527-534, 2012.

[18] K. Lu, J. M. Xu, S. Y. Chen, and L. Li, "A general model of bidirectional green wave for coordinate control of arterial road and its optimization solution," Control Theory Applications, vol. 28, no. 4, pp. 551-555, 2011.

[19] B. Cesme and P. G. Furth, "Self-organizing traffic signals using secondary extension and dynamic coordination," Transportation Research Part C: Emerging Technologies, vol. 48, pp. 1-15, 2014.

[20] J.-Q. Li, "Bandwidth synchronization under progression time uncertainty," IEEE Transactions on Intelligent Transportation Systems, vol. 15, no. 2, pp. 749-759, 2014.

[21] G. Gomes, "Bandwidth Maximization Using Vehicle Arrival Functions," IEEE Transactions on Intelligent Transportation Systems, vol. 16, no. 4, pp. 1977-1988, 2015.

[22] B.-L. Ye, W. Wu, and W. Mao, "A Two-Way Arterial Signal Coordination Method with Queueing Process Considered," IEEE Transactions on Intelligent Transportation Systems, vol. 16, no. 6, pp. 3440-3452, 2015.

[23] X. Yang, Y. Cheng, and G.-L. Chang, "A multi-path progression model for synchronization of arterial traffic signals," Transportation Research Part C: Emerging Technologies, vol. 53, pp. 93-111, 2015.

[24] C. Zhang, Y. Xie, N. H. Gartner, C. Stamatiadis, and T. Arsava, "AM-band: an asymmetrical multi-band model for arterial traffic signal coordination," Transportation Research, Part C: Emerging Technologies, vol. 58, pp. 515-531, 2015.

[25] S. Kim, A. Hajbabaie, B. M. Williams, and N. M. Rouphail, "Dynamic Bandwidth Analysis for Coordinated Arterial Streets," 
Journal of Intelligent Transportation Systems: Technology, Planning, and Operations, vol. 20, no. 3, pp. 294-310, 2016.

[26] G. Y. Dai, H. Wang, and W. Wang, "Signal optimization and coordination for bus progression based on MAXBAND," KSCE Journal of Civil Engineering, vol. 20, no. 2, pp. 890-898, 2016.

[27] M. J. Shirvani and H. R. Maleki, "Enhanced variable bandwidth progression optimisation model in arterial traffic signal control," IET Intelligent Transport Systems, vol. 10, no. 6, pp. 396405, 2016.

[28] L. Zhang, Z. Song, X. Tang, and D. Wang, "Signal coordination models for long arterials and grid networks," Transportation Research Part C: Emerging Technologies, vol. 71, pp. 215-230, 2016.

[29] H. Zhou, H. G. Hawkins, and Y. Zhang, "Arterial signal coordination with uneven double cycling," Transportation Research Part A: Policy and Practice, vol. 103, pp. 409-429, 2017.

[30] K. Lu, J. Hu, J. Huang, D. Tian, and C. Zhang, "Optimisation model for network progression coordinated control under the signal design mode of split phasing," IET Intelligent Transport Systems, vol. 11, no. 8, pp. 459-466, 2017. 


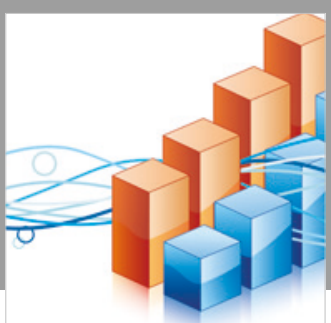

Advances in

Operations Research

\section{-n-m}
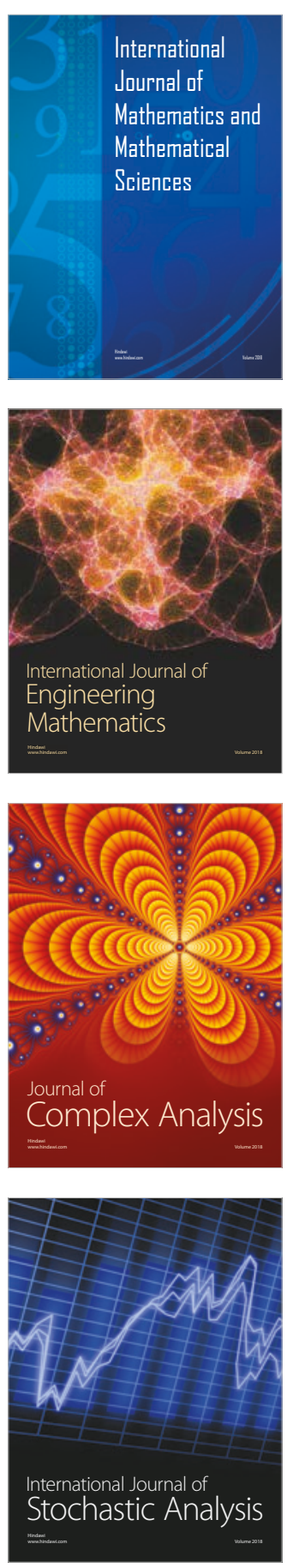
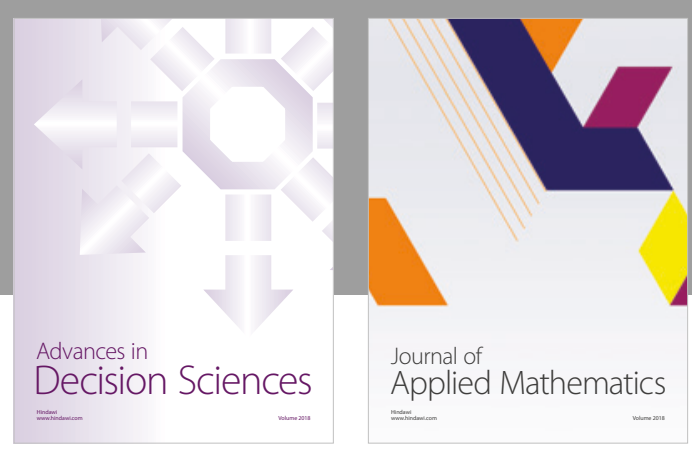

Journal of

Applied Mathematics
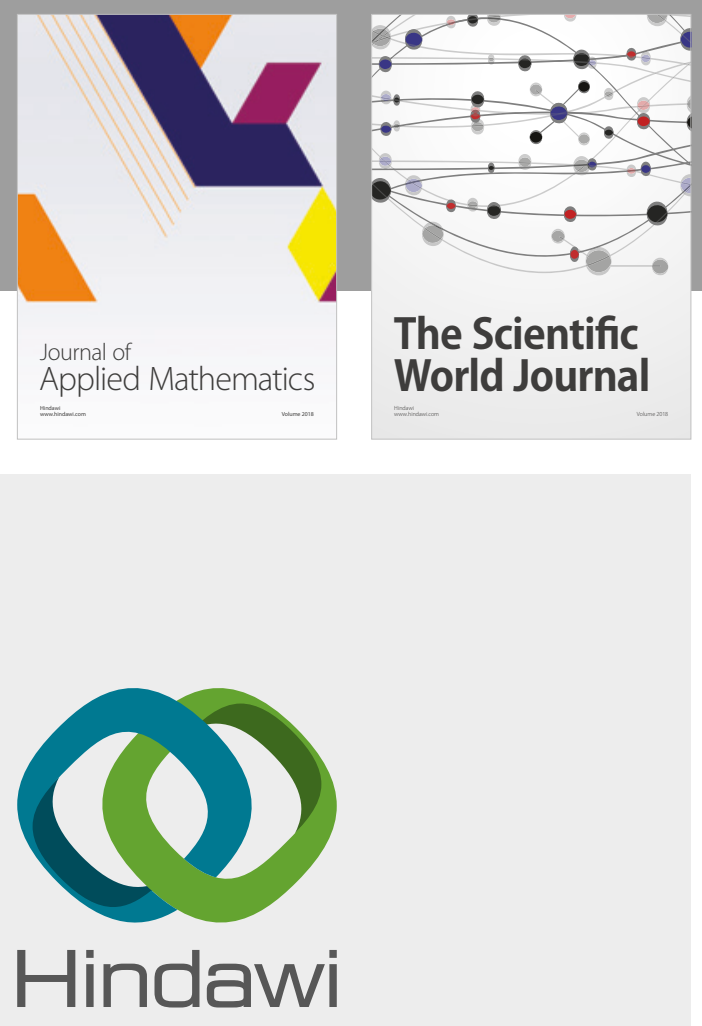

Submit your manuscripts at

www.hindawi.com

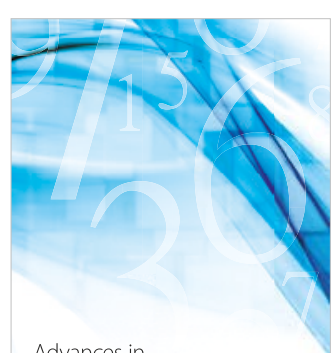

Advances in
Numerical Analysis
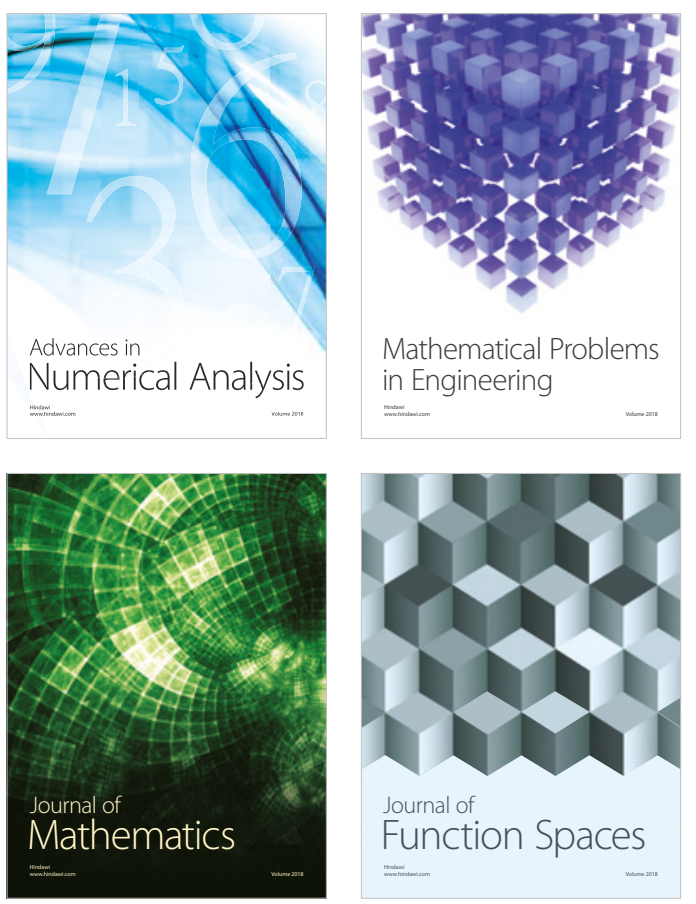

Mathematical Problems in Engineering

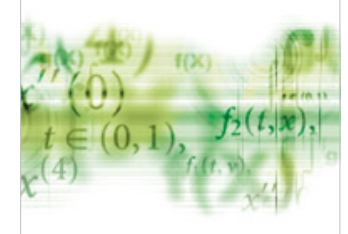

International Journal of

Differential Equations

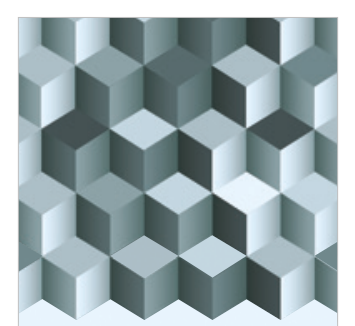

Journal of

Function Spaces

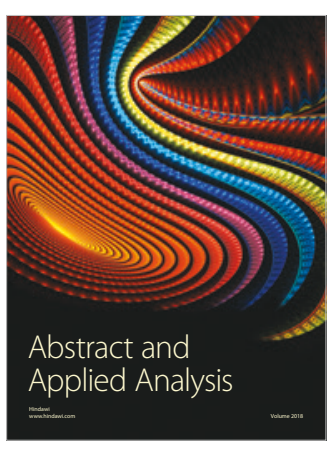

The Scientific

World Journal

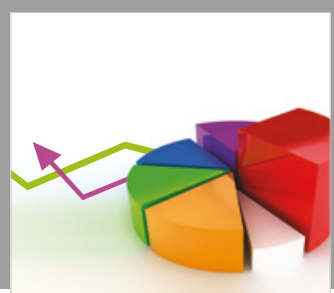

Journal of

Probability and Statistics
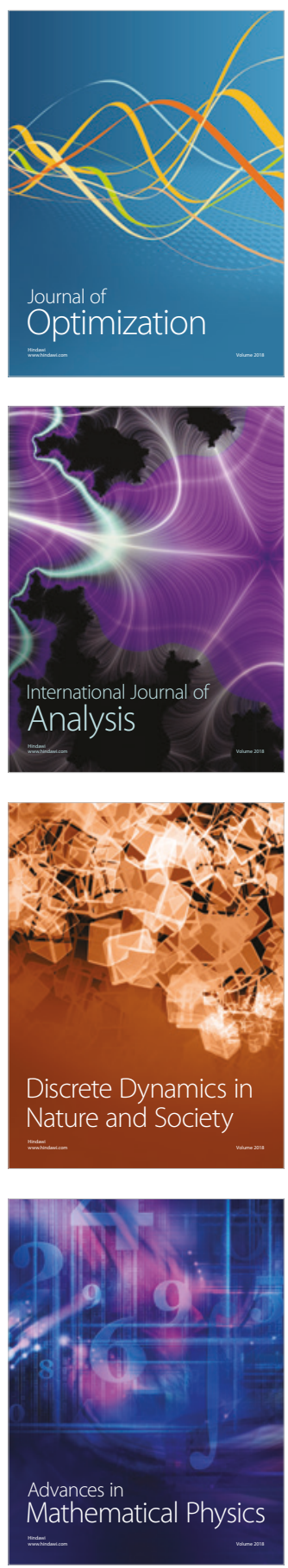